\section{Velcro compression devices}

\section{Joseph A. Caprini \\ University of Chicago, Pritzker School of Medicine, Chicago, IL, USA}

Leg swelling can occur from obstruction of the veins or venous reflux, resulting in increasing the volume of blood in the leg. This increases pressure in the capillaries causing them to dilate and, as this process continues, the endothelium cracks. The combination of slow flow in the capillary due to increased pressure and the endothelial damage causes white cells along with activated hemostatic elements to obstruct the capillary. No longer is there exchange of nutrients and waste products through the capillary membrane, so the area normally supplied by these capillaries becomes ischemic. Tissue necrosis may slowly occur resulting in skin ulceration.

Correction of leg swelling is not easy in many patients when the process is severe and has persisted for a long time. Selection of the proper compression modality is critical to achieve a successful result. Compression for this purpose includes both elastic and inelastic compression modalities. Elastic long-stretch compression is characterized by a high resting pressure, which may be uncomfortable over time. When the patient ambulates, a low ambulatory pressure results since the bandage gives way with walking and edema increases. ${ }^{1}$ The static stiffness index for elastic compression is less than $10 \mathrm{mmHg}$ and this may be defined as the difference between the under bandage pressure in the lying $v s$ standing position. ${ }^{2}$ Elastic bandages and stockings of various strengths are typical long-stretch products. They may suffice for mild swelling but are inadequate for patients with advanced leg edema. Inelastic or short-stretch compression products feature low resting pressure, which is comfortable at rest. As the patient stands and walks the pressure underneath the bandage increases, resulting in a decrease in edema as blood is pumped out of the leg. The rigid nature of the bandage produces this effect and the static stiffness index is greater than 10 for a good short-stretch compression product. Typical examples include a paste bandage (Unna's boot), short-stretch bandages and Velcro devices. The four- layer bandage consists of elastic products but when they are wrapped over one another they produce a similar effect to a good short-stretch bandage. A two -layer system is also an option. ${ }^{3}$

The initial compression treatment of the patient with leg swelling should consist of a good short-stretch product. When the edema is reduced, one of the best methods to maintain this effect and prevent recurrence is a proper Velcro device. These devices use short-stretch material which achieves a high working pressure and are quite comfortable at rest. As leg edema is further reduced with these devices the straps can be tightened to maintain a good compression effect. These products are usually worn over a light stocking or hybrid liner with foot compression only. In most cases the static stiffness index will exceed $20 \mathrm{mmHg}$ and may be much higher depending on the tightness of the device. ${ }^{4}$

Indications for these Velcro devices include patients having difficulty donning or doffing stockings due to advanced age, decreased hand and arm strength, the presence of arthritis or restricted mobility from obesity or advanced pregnancy. They are also very useful to prevent postoperative swelling after joint replacement as well as to reduce swelling in the preoperative patient. They are ideal for those suffering from lymphedema of the lower extremity. Patients with a large abdominal girth have increased venous pressure due to increased intra-abdominal pressure which may exceed $50 \mathrm{mmHg}$ in some patients. The standard 30 to $40 \mathrm{mmHg}$ stockings are unable to reduce swelling, pain and discomfort due to their elastic properties, and are extremely difficult to apply and remove especially in the obese patient. Poor compliance in these patients due to the above-mentioned factors results in further increased leg swelling, venous stasis and increased fragility. Finally, a point is reached when the stockings no longer even fit due to the increased size of the legs. Some patients in addition to using the Velcro devices on the calf, will require Velcro foot compression for swelling. Most patients with slight foot swelling can wear hybrid stockings under the calf Velcro device. These hybrid stockings are popular and feature $20 \mathrm{~mm}$ foot compression and negligible leg compression. Patients are encouraged to wear good, laced shoes to help control the swelling. ${ }^{5}$

Velcro devices are a good alternative to compression bandaging in some patients with leg ulcers. They have shown to be effective for ulcer healing and have several advantages compared to bandages. The patient can remove these devices daily for cleansing of the wound and dressing changes. One manufacturer has a unique system to adjust Velcro pressures using a simple measuring system. This device can be fitted in the office and tailored to the individual patient without the need for special fitting at an outside facility. This may represent an economic advantage for the
Correspondence: Joseph A. Caprini, University of Chicago, Pritzker School of Medicine, Chicago, IL, USA.

E-mail: jcaprini2@aol.com

This work is licensed under a Creative Commons Attribution 4.0 License (by-nc 4.0).

(C) Copyright J.A. Caprini, 2017

Licensee PAGEPress, Italy

Veins and Lymphatics 2017; 6:6624

doi:10.4081/vl.2017.6624

patient as well as the treating physician. Follow-up care once the ulcer is healed is simplified as long as the patient is compliant with wearing their Velcro devices. ${ }^{6}$

Mixed arterial and venous insufficiency occurs in at least $25 \%$ of patients and these individuals have reduced ankle pressures as well as swollen legs. Use of elastic products on these legs may be hazardous due to the high resting pressure which may further compromise the leg circulation. Velcro compression devices are ideal for this type of patient as they provide a low resting pressure and high working pressure. They are easily adjusted to tighten or loosen depending on the patient's tolerance and symptoms such as pain, numbness or tingling. They can be used as long as the resting pressure of the Velcro device is less than the systolic pressure at the ankle. Arterial inflow may actually increase due to the decrease in venous volume and pressure of the leg allowing improved arterial perfusion. ${ }^{7}$

Patients requiring major arterial revascularization such as open femoral popliteal bypass procedures often suffer from reperfusion leg edema. This swelling can result in breakdown of the incisions leading to infection and eventual graft rejection. The use of Velcro devices in these situations is an ideal way to control the swelling in a safe manner without compromising the newly re-vascularized limb.

Patients requiring major leg resection for cancer often suffer from significant swelling including lymphedema after lymph node dissection. Velcro devices are a very valuable adjunct for these individuals. Another important management problem occurs in patients who have life-threatening leg infection requiring major resection of the tissues. The long-term control of healed wounds and leg swelling is greatly facilitated using Velcro devices.

In conclusion, we feel it is very important for practitioners to understand the basic principles concerning leg swelling and the characteristics of different compression products. Practitioners dealing with these patients should learn when stockings are not 
appropriate and be familiar with the shortstretch products that may achieve success. Velcro compression appliances should be considered in patients who fail standard support stockings. Some patients who should not be prescribed stockings include noncompliance due to donning or doffing issues, failure to control swelling, morbid obesity, and patients with arthritis and decreased flexibility which may occur with advanced age. Patients with mixed arteriovenous insufficiency are excellent candidates for these devices as are those having joint replacement or major leg surgery for infection or cancer. They may also be effective as an alternative treatment to compression bandages for selected leg wounds.

\section{References}

1. Mosti G, Mattaliano V, Partsch H. Inelastic compression increases venous ejection fraction more than elastic bandages in patients with superficial venous reflux. Phlebology 2008;23:287-94.

2. Partsch H. The static stiffness index- a simple method to assess the elastic property of compression material in vivo. Dermatol Surg 2005:625-30.

3. Mosti G. Comparing 2 bandages in ulcer healing. Wounds 2011;23:126-34.

4. Mosti G, Mattaliano V, Partsch H. Inelastic compression increases venous ejection fraction more than elastic band- ages in patients with superficial venous reflux. Phlebology 2008;23:287-94.

5. Mosti G, Cavezzi A, Partsch H, et al. Adjustable velcro compression devices are more effective than inelastic bandages in reducing venous edema in the initial treatment phase: a randomized controlled trial. Eur J Vasc Endovasc Surg 2015;50:368-74.

6. DePalma RG, Kowallek D, Spence RK. Comparison of costs and healing rates of two forms of compression in treating venous ulcers. J Vascular Surgery 1999;33:683-90.

7. Mosti G. Compression in mixed ulcers. Veins and Lymphatics 2016;5:5986. 